
internationales

vol. 30 - n² | 2014

Composer (avec) la frontière. Passages, parcours migratoires et échanges sociaux

\title{
Vivre sur, vivre de la frontière : l'après transit en Mauritanie et au Mali
}

Living on the Frontier: Post-transit in Mauritania and Mali

Vivir en, vivir de la frontera: el postránsito en Mauritania y Mali

Jocelyne Streiff-Fénart et Philippe Poutignat

\section{(2) OpenEdition}

\section{Journals}

\section{Édition électronique}

URL : https://journals.openedition.org/remi/6911

DOI : 10.4000/remi.6911

ISSN : $1777-5418$

\section{Éditeur}

Université de Poitiers

\section{Édition imprimée}

Date de publication : 1 juin 2014

Pagination : $91-111$

ISBN : 979-10-90426-22-1

ISSN : 0765-0752

\section{Référence électronique}

Jocelyne Streiff-Fénart et Philippe Poutignat, «Vivre sur, vivre de la frontière : I'après transit en

Mauritanie et au Mali », Revue européenne des migrations internationales [En ligne], vol. 30 - n² | 2014, mis en ligne le 01 juin 2017, consulté le 16 avril 2022. URL : http://journals.openedition.org/remi/6911 ; DOI : https://doi.org/10.4000/remi.6911 


\section{Vivre sur, vivre de la frontière : l'après transit en Mauritanie et au Mali}

\section{... Jocelyne Streiff-Fénart ${ }^{1}$ et Philippe Poutignat ${ }^{2}$}

Cet article porte sur la situation des migrants " de transit " qui sont restés dans le pays traversé, en prenant comme lieux d'observation les villes de Nouadhibou en Mauritanie et Bamako au Mali dans lesquelles nous avons eu l'occasion de mener des enquêtes prolongées, permettant le suivi des trajectoires migratoires. Au cours de séjours annuels successifs depuis 1995 en Mauritanie et 2008 au Mali, nous avons recueilli un très vaste corpus d'histoires de vie, de récits de voyage, de discussions de groupes et mené sur chaque terrain des observations in situ des interactions entre les migrants ou entre eux et les autres acteurs: ONG, organismes de gestion des migrations, membres des sociétés locales, voisins, propriétaires, employeurs. C'est à partir de la confrontation de ces points de vue, des tensions qui les traversent, des prescriptions normatives ou des revendications de reconnaissance qu'ils expriment, que nous avons tenté d'appréhender la spécificité de l'expérience migratoire hybride de ceux qui ont cessé d'être des " aventuriers " ${ }^{3}$ sans pour autant devenir à proprement parler des immigrés.

Précisons tout d'abord que la notion de "transit " appliquée à certains lieux et à certains types de migrants ne trouve de sens que dans la vision euro-centrée de routes migratoires polarisées par leur destination, qui s'est imposée depuis la mise en place du dispositif de surveillance des frontières extérieures de l'Europe. Avec l'externalisation du contrôle, incluant des zones situées dans des pays et des continents différents, certains pays (ceux notamment du Maghreb, mais aussi tous ceux qui " accueillent " les expulsés et refoulés) sont considérés comme des espaces-frontières, non pas en vertu d'une ligne de démarcation séparant de façon nette deux territoires, mais par l'anticipation du franchisse-

\footnotetext{
1 Directrice de recherche CNRS, URMIS (CNRS UMR 8245 - IRD UMR 205 - Universités Nice Sophia Antipolis et Paris Diderot), MSHS Campus Saint Jean d'Angely, 3 boulevard François Mitterrand, 06357 Nice cedex 4 ; streiff@unice.fr

2 Chercheur CNRS, URMIS (CNRS UMR 8245 - IRD UMR 205 - Universités Nice Sophia Antipolis et Paris Diderot), MSHS Campus Saint Jean d'Angely, 3 boulevard François Mitterrand, 06357 Nice cedex 4 ; poutigna@unice.fr

3 Sur ce terme et ses usages dans différents contextes, voir Pian, 2008 ; Bredeloup, 1994 et 2008 ; Poutignat et Streiff-Fénart, 2006.
} 
ment potentiel d'une telle ligne située plus au nord. Les migrants de transit sont alors ceux qui, bloqués dans leur tentative d'atteindre l'Europe sans disposer de visas, s'attardent dans ces espaces en attente d'une prochaine occasion de tenter le passage.

Il est important de relever que les effets du contrôle migratoire ne consistent pas seulement à empêcher le franchissement d'une frontière physique, mais aussi à établir une frontière morale, celle qui sépare les mobilités vertueuses (celles des touristes, hommes d'affaires ou immigrés réguliers) des déplacements frauduleux, clandestins, illégaux, bref immoraux, des vagabonds (Bauman, 1999). Le pouvoir qu'exercent les institutions en charge du contrôle de ces mobilités avec son cortège de drames et de violence (noyades, traversées mortelles du désert, viols, dépouillements, enfermement) a une forte dimension $\mathrm{d}^{\text {'arbitraire }}{ }^{4}$ et donc une forte exigence de justification dont témoignent le riche répertoire de mots (suicide, inconscience, eldorado, ruée, trafic, mafia) et l'arsenal de mesures (campagnes de dissuasion, aide au "retour volontaire ") mettant l'accent sur le caractère à la fois contraint et irrationnel de ces mobilités.

Cette présentation des migrants comme les victimes des passeurs, de I'avidité de leurs familles ou de leur propre folie (largement relayée par les médias) s'appuie sur un cadrage préalable de la mobilité comme problème, comme indice d'une crise, quand ce n'est pas d'un fléau, et renvoie plus généralement à une représentation de la stabilité comme norme ${ }^{5}$ (Bakewell, 2008).

Ce qui ressort nettement des études de terrain, c'est que précisément cette façon de penser la migration et les migrants comme problème est assez étrangère aux opinions publiques dans les deux villes sur lesquelles nous avons mené nos enquêtes ${ }^{6}$. Ce fait est d'ailleurs déploré par les agents des organismes internationaux chargés de la mise en œuvre d'une " bonne gouvernance " des migrations, qui le mentionnent spontanément lorsqu'ils évoquent les difficultés rencontrées dans leur pratique professionnelle. Par exemple, le représentant de I'Organisation Internationale pour les Migrations (OIM) à Nouakchott, lors d'un entretien en 2009, tient à nous signaler d'emblée comme un problème marquant que la question des migrations ne fait pas l'objet de "débats émotionnels " en Mauritanie. Personne, déplore-t-il, " ne remet en cause le statu quo sur les facilités de voyage des migrants" ".

La compréhension de la migration comme anormale et problématique ne va donc pas de soi, y compris chez les partenaires locaux des organismes

\footnotetext{
4 Comme le soulignent les associations de défense des migrants, le droit de quitter son pays est une liberté garantie par les législations internationales et il est moralement difficile de dénier à tout un chacun le droit de fuir la misère ou les violences dans son pays.

5 La notion de " retour volontaire " concentre ces dimensions normatives, ce qui explique sans doute qu'elle soit érigée en modèle par les organismes internationaux en dépit du nombre dérisoire d'actions de ce type en réalité. Le retour volontaire s'oppose à l'expulsion comme deux modalités de retour à la norme (rester chez soi), répondant à deux façons de la transgresser : par un acte pleinement assumé (qui n'est réparable que par la contrainte : I'expulsion) ou par une défaillance cognitive (réparable par l'explication, l'information et la persuasion, donc accessible aux actions dissuasives).

6 Tout au moins jusqu'à une date récente pour ce qui concerne Nouadhibou, où comme on le note plus loin, la situation a évolué de ce point de vue.
} 
internationaux présents sur le terrain. Lors de nos entretiens avec des responsables de ces organismes, il était courant que les agents locaux assistant à l'entrevue (depuis le chargé d'étude jusqu'au représentant de l'État) manifestent leur perplexité devant I'hypothèse implicite que la sédentarité est la situation normale et désirable pour les humains. Par exemple, lors d'un entretien à Bamako au siège de I'OIM en 2009 :

" [Le représentant de I'OIM :] - "Jusqu'ici les pays africains ne voient pas la migration comme un problème, mais comme un phénomène normal ".

[Le chargé d'étude malien recruté pour établir les "profils migratoires" intervient pour remarquer que les Maliens ont eux-mêmes une grande habitude des migrations et que donc ils comprennent les migrants et ils ne sont pas contre].

[Le représentant de l'OIM :] - "Mais si ça n'a pas éclaté comme problème dans l'opinion publique, les migrants néanmoins ont des problèmes et des besoins" ".

Cet échange est tout à fait typique d'un procédé discursif qui participe du processus de justification : la flexibilité de l'idée de problème associée aux migrants de transit, comme avoir un problème (le problème des migrants en transit) ou être un problème (le problème causé par les migrants en transit). Flexibilité qui a des implications importantes, puisqu'elle renvoie à (et autorise) un traitement des migrants, fondé sur l'ambiguïté entre logique sécuritaire (le problème par) et logique assistantielle (le problème de).

La politique européenne d'endiguement des flux et les élaborations discursives qui la mettent en forme ont imposé l'évidence d'un phénomène, la migration de transit, que cette même politique fait advenir à la réalité. Catégorie créée par les organismes étatiques et internationaux en charge de la gestion des flux migratoires, la migration " de transit " n'en représente pas moins pour les migrants ainsi désignés une expérience migratoire spécifique, celle qui, contrairement au sens courant du " transit " comme point de passage et moment furtif, installe le voyageur sur un seuil (Streiff-Fénart et Segatti, 2011). Dans ces lieux où l'on est arrêté en chemin, certains ne font que passer tandis qu'ils deviennent pour d'autres des lieux de vie sans qu'ils veuillent pour autant, ni qu'ils ne soient appelés à s'intégrer ${ }^{7}$.

\section{S'installer sur la frontière}

Les migrants dont nous rapportons ici les trajectoires sont généralement désignés comme " aventuriers " ou "clandestins", comme c'est le cas dans la ville portuaire de Nouadhibou qui a connu au milieu des années 2000 un afflux de migrants en quête du passage vers les Canaries. Au Mali, ils sont plutôt identifiés sous l'appellation de " refoulés ". On désigne ainsi localement les migrants étrangers expulsés vers le Mali depuis l'Algérie ou la Mauritanie, qui constituent dans la capitale ou dans les villes du Nord proches de la frontière algérienne des " communautés d'itinérance " (Escoffier, 2008), clairement distinctes des communautés de migrants de même origine nationale (Camerounais, Ghanéens, Ivoiriens, etc.) anciennement installées à Bamako.

7 Pour des descriptions détaillées de la situation des migrants de transit au Maroc, voir Goldschmidt, 2002 ; Alioua, 2005 ; Pian, 2008 ; Timéra, 2009. 
Les parcours migrants que nous rapportons ci-dessous s'inscrivent tous dans cette forme originale d'émigration que décrit le terme d'" aventure ", originale en tant qu'elle constitue une expérience individuelle de l'acteur de sa propre migration qui s'" aventure " tout seul, contrastant ainsi avec le système d'action qui prévalait dans les " migrations ordonnées " du premier âge décrites par Sayad (1977). L'idéal individualiste de réalisation de soi, voire d'accomplissement du sens de son existence (Timéra, 2009) qui caractérise ces mobilités, n'implique pas pour autant qu'elles soient le fait de sujets détachés des contraintes et des devoirs familiaux que I'on attend des émigrés. Dans la plupart des cas, les familles ont participé pour tout ou partie au financement du voyage et escomptent le remboursement de la dette tout à la fois financière et sociale contractée par l'aspirant au départ. Les migrants de l'aventure sont pris dans une double contrainte : I'interdiction de migrer par les États, sanctionnée par l'expulsion ou le refoulement, et l'injonction à la réussite du projet migratoire exercée par les familles, sanctionnée par l'ostracisation du migrant en cas de retour dans l'échec.

C'est précisément le processus de subjectivation lié à la fois à l'immobilisation imposée par le dispositif du contrôle des frontières et aux stratégies de prise de distance avec les contraintes du milieu d'origine, qui rend ces mobilités accessibles à l'analyse en tant qu'expérience sociale spécifique (Poutignat, 2011). Restituer cette expérience implique de centrer l'analyse sur la compréhension que les acteurs migrants ont de leur propre trajectoire, en tenant à distance les représentations conventionnelles qui les réifient comme des victimes de trafics, des vagabonds misérables ou des nomades créatifs.

En restant au plus près des récits de vie recueillis, on peut constater que ce qui caractérise au premier chef la migration d'aventure, c'est l'imprévisibilité de la vie sociale et l'absence de maîtrise sur l'horizon temporel des projets. Si bien que le sens même de l'aventure se forme en situation, dans la succession d'épreuves par laquelle s'apprivoise "la chance ${ }^{8}$, cette divinité capricieuse des aventuriers.

Dans ces situations d'entre-deux où rien n'est joué d'avance, toute réussite, même relative, en résorbant l'incertitude, vaut justification du cours d'action entrepris. C'est ce que nous explique ce migrant nigérian attentif aux signes de la grâce divine qui lui permettent d'établir de façon rétrospective et prospective la cohérence de ses expériences:

"La vie est une chance, un destin. [...] Si Nouadhibou est ma destinée, je le saurai sûrement, parce que je vais progresser dans ce que je fais. [...] Peut-être maintenant je suis en route pour l'Europe, pour chercher l'argent, mais l'argent, si j'en gagne beaucoup en six mois, je serai convaincu de ne pas partir. Je vais commencer une affaire, elle va progresser [...] ; je commencerai à améliorer ma situation [...]. Je sais que c'est mon destin lorsque ma vie a changé. [...] Vous pouvez dire c'est le destin dans un pays quand vous faites des progrès " (Jonathan, Nouadhibou, décembre 2005).

8 Tous les mots placés entre guillemets renvoient à des expressions récurrentes dans les entretiens. 
Les lieux dans lesquels se déploient les itinéraires d'après transit qui font l'objet de cet article sont ceux où, pour reprendre une expression récurrente dans les récits, on a "trouvé sa chance ", sa "destinée ", où, pour ceux qui se définissaient comme des " aventuriers ", s'opère une sorte de métamorphose sociale : le changement du sens du voyage. Souvent décrits comme piégés dans une situation de nasse ou un cul-de-sac (Pian, 2009 ; Ba et Choplin, 2005), les migrants qui s'attardent dans ces lieux où ils sont arrêtés ou refoulés y trouvent parfois une niche, une opportunité d'emploi qui les conduit à différer leur projet, voire à y renoncer. Même s'il reste toujours perçu comme provisoire, l'ancrage dans un lieu qui n'est ni celui du départ ni celui de la destination visée permet de reprendre le fil de la vie interrompu par l'aventure. Lieu de dénuement, où I'on souffre, mais qui vaut précisément par cette souffrance, si bien que le quitter signifierait que les sacrifices consentis pour " se refaire " ont été faits en pure perte.

Nouadhibou et Bamako sont des lieux de transit que les aventuriers qui s'y attardent se sont dans une certaine mesure, appropriés, où l'on a ses amis, ses habitudes, son réseau institutionnel. Même si l'on y vit mal, il n'est pas question d'aller dans un autre pays d'Afrique où " il faudrait tout recommencer à zéro ".

Dans les deux villes, la pluriethnicité et la mobilité sont des traits inhérents à la vie urbaine : déplacements périodiques des urbains entre ville et village, migrations transfrontalières avec les pays voisins, réseaux marchands de proximité ou à longue distance, etc. Les migrants nouvellement arrivés y trouvent des communautés de compatriotes établis de plus longue date et des relais migratoires (foyers, associations, réseaux familiaux). II existe des échanges et une certaine porosité entre ces différentes strates de la migration, liées par des relations souvent ambivalentes, oscillant entre solidarité et exploitation, sentiment communautaire et méfiance. Les " migrants de l'aventure " tirent toutefois de leur itinéraire spécifique, marqué par la prise de risque et la détermination à déjouer les obstacles, des ressources et des compétences leur permettant $d^{\prime}$ exploiter la frontière comme un espace $d^{\prime}$ action. $C^{\prime}$ est par exemple le cas de certains de ces migrants qui renoncent à leur projet personnel de passage en Europe pour se professionnaliser dans des fonctions du passage pour les autres (thiaman d'un ghetto ou passeur) (Laacher, 2007). Les observations qui suivent rendent compte d'autres façons de tirer profit de la vie sur la frontière. Si les itinéraires présentés sont fort différents, ils ont en commun de se brancher sur des réseaux transnationaux : un dispositif de commerce à longue distance, un réseau religieux, la nébuleuse des organismes de gestion, d'accueil et $d^{\prime}$ assistance aux migrants.

\section{Itinéraires d'après transit à Nouadhibou}

En dépit de la longue habitude de la présence des migrants, la question de l'intégration des étrangers ( $c$ 'est-à-dire celle qui consiste à faire la part entre bons et mauvais étrangers) est, en Mauritanie, une toute nouvelle question qui a émergé en même temps que les pratiques d'enregistrement et de tri des populations (campagne d'enrôlement, délivrance de cartes de résidence et de visas 
biométriques) et les objectifs affichés de mauritanisation des emplois ${ }^{9}$. Mais, c'est dès 2008, avec l'intensification du dispositif de surveillance des frontières mis en place sur ce point de passage ayant entraîné l'arrêt des départs des pirogues vers les Canaries, que les relations entre les locaux et les étrangers subsahariens ont commencé nettement à se détériorer (Choplin, 2010 ; Streiff-Fénart, 2011). Le ralentissement des départs sous l'effet du durcissement des contrôles n'ayant pas amené un relâchement des pressions de I'UE, la Mauritanie a poursuivi sa stratégie d'extraversion (Bayart, 1989) jouant de la conditionnalité des aides. Pour demeurer le " bon élève " qu'elle prétend être, en l'absence de flagrants délits, c'est désormais les arrestations préventives (rafles dans les foyers, arrestations dans la rue la nuit) qui deviennent la règle, se multipliant jusqu'à trouver leur apogée dans une véritable chasse aux " clandestins ${ }^{10}$.

Jusqu'alors, la situation des étrangers n'était pas particulièrement visée par des discours ou pratiques xénophobes ${ }^{11}$ pas plus qu'elle ne faisait l'objet de proposition d'intégration. Sans être à proprement parler accueillante, la société maure ménageait aux étrangers des niches d'emploi dans des secteurs économiques peu investis, en particulier dans sa capitale économique, permettant à ceux qui avaient l'habileté de s'en saisir des opportunités de prendre place.

\section{Adam : les hauts et les bas d'une carrière d'entrepreneur sur la frontière}

Le récit d'Adam, un Ivoirien arrivé en 2002 à Nouadhibou pour tenter le passage, montre comment un itinéraire de réussite pouvait être à portée de ceux qui savaient se saisir de ces opportunités. On peut aussi y voir la sinuosité de ces parcours de réussite, toujours pris dans des hauts et des bas, entre des périodes d'accumulation et des déboires divers : ceux qui sont le lot des étrangers dans une société qui ne leur offre aucune protection, mais aussi ceux qui résultent des mésententes familiales sur l'usage de leurs remises.

Adam, dont la famille est établie dans une ville du sud-ouest de la Côte $d^{\prime}$ Ivoire, a sept frères et sœurs, de même mère, et deux demi-frères. Il entretient une relation privilégiée avec une sœur aînée qui travaille au Burkina chez qui il a habité pendant une partie de ses études. Après une formation d'ébéniste, il a travaillé dans l'atelier de menuiserie d'un frère aîné avec qui il ne s'entend pas.

9 Se concrétisant, par exemple, par l'instauration en 2013 d'un nouveau permis qui exclut de fait les chauffeurs de taxi étrangers, jusque-là très nombreux dans la profession.

10 Lors de notre séjour en juin 2013, tous les anciens " aventuriers ", installés maintenant depuis de nombreuses années, nous font part des rafles systématiques dont ils ont été les victimes, à l'occasion de la mise en place de la carte de résidence pour les étrangers en 2012. Selon les récits, les " ramasses " ont été faites par la gendarmerie venue de Nouakchott (et non pas par la police locale), de façon totalement inopinée, et avec un caractère raciste (seuls les étrangers noirs étaient visés). Elles se sont déroulées tout au long d'une journée en avril, les étrangers arrêtés étant placés dans le centre de rétention rouvert pour l'occasion, puis dans la nouvelle prison, pas même encore inaugurée. Certains étrangers qui n'avaient pas leurs papiers " en règle " ont été expulsés vers le Sénégal, d'autres sont partis vers d'autres destinations. Les plus installés, pouvant dégager la somme nécessaire (30 000 UM par an et par personne) ont demandé la carte.

11 Le racisme et sa dénonciation étant plutôt centrés dans les débats publics sur la division interne entre Maures et Négro-Africains. 
Ayant entendu parler de Nouadhibou comme point d'accès à l'Europe, il prend la décision de s'y rendre avec l'approbation de sa famille, mais constate en y arrivant qu'il ne peut passer par la route. II informe immédiatement sa famille :

"Le premier jour, j'ai appelé ma grande sœur, je lui ai dit: "La situation n'est pas comme on le croyait, je suis bloqué en Mauritanie, appelle les parents". Elle m'a demandé : "Pourquoi les informer ? Attends! ". J'ai dit: "Non, non" " (Adam,

Nouadhibou, décembre 2005).

Afin d'éviter que les " malédictions " ne se multiplient, il est soucieux, en effet, qu'on ne le croit pas en Europe, oublieux de "la promesse " que constitue son départ. Le travail d'ébéniste rapportant peu à Nouadhibou, il trouve très vite le chemin de Bûntya ${ }^{12}$, un lieu réservé à la transformation artisanale du poisson, où les étrangers sont facilement embauchés, à la journée, pour l'écaillage du poisson. Pressé de rembourser I'hospitalité d'un Malien rencontré par hasard et qui l'a hébergé le premier mois, il accepte ce travail très dur, qu'évitent les quelques Ivoiriens sur place qui privilégient les emplois de chauffeurs ou d'enseignants de français. À Bûntya, d'abord employé comme contrat-man, payé à la tâche, il découvre progressivement l'organisation du travail qui y règne et quitte les emplois les plus mal payés, pour pouvoir commencer à économiser. Au bout des six premiers mois, ayant réuni environ 150 euros, il peut, tout en restant salarié, expédier ses premières caisses de guedj $j^{13}$ au marché de Nouakchott, imitant en cela de nombreux aventuriers qui cherchent, par ce moyen, à réunir le montant du passage. II réalise assez vite qu'il peut faire suffisamment d'argent sur place, et que l'Europe n'est pas un but en soi. II se donne alors cinq ans, qu'il qualifie de " sacrifiés " (c'est-à-dire de sacrifices) pour réussir.

Entre-temps, il a suffisamment appris les différents métiers présents sur le site pour s'établir comme transformateur de raies et requins en passant des contrats avec des opérateurs ghanéens qui financent ses achats auprès des mareyeurs. À partir de l'année 2004, soit deux ans après son arrivée, il commence à faire suffisamment d'économies pour envoyer de l'argent à sa famille, laquelle cependant comprend mal son choix et l'incite toujours à partir. Ses affaires changent d'échelle. Les quantités et les sommes en jeu sur le marché tenu par les Ghanéens sont importantes, mais si l'activité offre des possibilités spéculatives, elle comporte aussi des risques. En effet, les commandes sont à terme alors que les cours fluctuent selon les saisons tout en étant également soumis à des impondérables. Cela implique un nouveau type d'apprentissage : il faut s'assurer de la ressource en haute saison, jouer sur le crédit en basse saison, être en mesure autant que possible d'avoir ses propres stocks, avec le risque que la marchandise se gâte. Il faut enfin se garantir en diversifiant ses activités sur plusieurs marchés, ce qu'il fait en prenant également les commandes de Nigérians de passage et surtout en continuant la production de guedj que sa femme togolaise, épousée sur place, commercialise à Nouakchott. Sur ce marché, Adam parvient à s'imposer comme un opérateur fiable à l'égard de ses commanditaires. II est un bon payeur qui honore toujours sa parole, même lorsqu'il est perdant. II sait aussi, dans un marché largement informel,

12 Pour des descriptions détaillées de ce lieu, des activités qui s'y déroulent, des circulations commerçantes qui s'y croisent, voir Poutignat et Streiff Fénart, 2006.

13 Poisson fermenté séché à destination du marché sénégalais, malien ou mauritanien. 
jouer de l'écriture pour faire valoir ses droits. C'est à la fois un chef d'entreprise (il emploie plusieurs contrat-men maliens) et un homme d'affaires, sans cesse en train de calculer, le portable à l'oreille, pour être informé des arrivages au port et donner ses ordres. Il expédie tous les trois mois 150 euros à sa mère et pour faire parvenir de l'argent au pays et éviter les frais de change, il lui arrive de confier des cargaisons de guedj à un commerçant sénégalais qui tourne entre le Mali, la Côte d'Ivoire et le Sénégal.

Au bout de cinq ans, il estime disposer d'un capital monétaire d'environ 10000 euros. II hésite à investir dans la menuiserie de son frère qu'il juge mal gérée. Il fait le projet de deux restaurants au Burkina, dont l'un sera géré par sa sœur et l'autre par lui. II fera venir un petit frère et pourra étendre ses affaires, à partir du Burkina, sans plus sacrifier sa vie. II retarde ce projet plusieurs fois pour faire grossir encore son capital et finalement s'en va. II passe en Côte d'lvoire où il investit une partie de son argent dans l'achat de onze parcelles à bâtir dans une visée spéculative. Par ailleurs, la mauvaise opinion qu'il a de ses frères trouve à se confirmer. II leur a expédié de l'argent pour investir dans la maison familiale afin de la rénover et de l'agrandir pour en faire un immeuble de location et ouvert un compte spécial destiné à recueillir les loyers. Au lieu de faire ces placements, ils se sont contentés de dépenser l'argent, pour leurs propres besoins. Avec l'accord du chef de lignage, il convoque un conseil de famille qui retire la gestion de l'argent à ses frères et désigne un de ses amis d'enfance comme nouveau gestionnaire de ses envois d'argent. Ainsi, la réussite de la migration d'Adam est-elle officialisée d'une façon qui ne laisse pas inchangée l'ordre communautaire et familial qu'il a réintégré : d'une part sa souscription à l'économie morale de la dette (Marie, 1997) se traduit par des investissements immobiliers (plutôt que par la participation aux entreprises de ses frères), d'autre part le souci de ne pas dilapider l'argent, entérine en sa faveur le réaménagement de l'ordre hiérarchique en court-circuitant les frères aînés. Les conditions ont été ainsi créées pour qu'Adam puisse voir reconnues à la fois son autonomie et son inscription dans l'ordre communautaire.

Mais la poursuite de son parcours personnel devait se dérouler au Burkina avec les parents dont il se sent le plus proche, sa sœur aînée et son petit frère. Or, une fois sur place, le projet initial tourne court. À la place des deux restaurants prévus, il n'y a qu'un seul restaurant-bar à se partager. La mauvaise entente entre sa femme et sa sœur, avec ses risques de sorcellerie, le contraint à tout laisser sans contrepartie. Sans autres moyens, il se tourne alors vers un gros client ghanéen, qui l'avait invité à visiter son pays. Ce dernier est disposé à travailler de nouveau avec lui. Pour se maintenir dans sa trajectoire d'accumulation économique et $d^{\prime}$ ascension sociale, et ne pas gaspiller les savoir-faire et le capital relationnel acquis, Adam doit alors retourner à Nouadhibou. II y retrouve son atelier et se donne un nouveau délai, deux ou trois ans de sacrifices encore, pour réunir le capital lui permettant de mener à bien ses projets immobiliers. En 2009, il se fait cambrioler à son domicile la somme confiée par son commanditaire ghanéen pour l'achat du poisson, somme qu'il se sent obligé de rembourser intégralement en puisant dans ses économies pour préserver sa réputation d'honnêteté, capital précieux sur ce marché. Nouveau déboire qui le maintient quelques années supplémentaires en Mauritanie, entre Nouadhibou et Nouakchott où il se trouvait encore à notre dernier passage en 2013. 


\section{Guillaume : une figure de l'aventurier simmelien}

Le récit de Guillaume, un Ivoirien rencontré à la mission catholique de Nouadhibou en 2005, illustre une autre expérience d'installation sur la frontière, d'autant plus singulière dans son cas qu'elle s'extrait de la nécessité économique qui est la toile de fond de l'expérience sociale de la migration. Ayant su saisir les occasions qui se présentent à lui, mais aussi en restant fidèle à l'idée qu'il se faisait de sa vie, son parcours réalise le type de synthèse entre la contingence d'une situation et la nécessité intérieure, qui définissait pour Simmel (2002) l'aventure.

Pour expliquer ce qui I'a amené à sortir et à continuer le voyage, il ne trouve pas de meilleure raison que son goût pour les études. À ce moment, il occupe déjà une petite pièce au bout du bâtiment de la mission catholique. Aux horaires de bureaux, il tient à jour les fiches des aventuriers encouragés à s'inscrire pour qu'une trace soit gardée de leur passage alors que commencent à partir les pirogues et à s'échouer les naufragés. C'est un travail de confiance, presque bénévole, matériellement une sorte de dépannage, mais qui lui convient. II a accès à Internet, un privilège ici, et aux livres de la bibliothèque. II connait nécessairement beaucoup de monde, la mission est un de ces lieux de rassemblement et d'accueil qui amène à visibilité " la communauté d'itinérance " des aventuriers qu'il peut réintégrer à tout moment, mais dont la fonction qu'il occupe le sépare quelque peu. Cette position pour modeste qu'elle soit, n'est pas sans prestige ; elle est un premier accomplissement, mais elle n'est peut-être aussi qu'une étape, une position d'attente, un " entre-deux ", dans l'enclave accordée à l'altérité que représente la mission.

" Jusqu'à présent, j'ai l'idée de vouloir partir, mais c'est pas parce que je suis à Nouadhibou que je suis là pour prendre la pirogue ; [...] peut-être qu'avec le temps, peut-être que l'occasion peut se présenter, peut-être un jour... On ne sait jamais ce qui nous pousse à tel moment de prendre telle ou telle décision " (Guillaume, Nouadhibou, décembre 2006).

Sa famille, malgré sa pauvreté, lui a permis de poursuivre les études jusqu'au bac. Les études lui ont toujours plu, il n'a jamais échoué :

" Je voulais aller le plus loin possible, avoir un très bon niveau, je me disais, je peux, je peux, je peux... " (Guillaume, Nouadhibou, décembre 2006).

Après son bac technique, la poursuite d'études supérieures compromettrait la scolarisation de ses cadets (il est l'aîné de cinq enfants). II cherche à entrer dans la vie active sans succès et, avec un ami d'enfance qui a eu le même parcours scolaire que lui, ils se préparent à "sortir " en accumulant les conseils et en comparant les itinéraires détaillés qu'ils trouvent autour d'eux. Ils avertissent leur famille au dernier moment pour recevoir leur bénédiction, quittent la Côte d'Ivoire fin 2000, pour se retrouver plus tard bloqués à Agadez, où ils connaissent la pire misère. Ils arrivent à survivre, rebroussent chemin à Niamey, parviennent à joindre leur famille qui leur envoie un peu d'argent et les incitent à revenir. 
"On s'est dit: on va essayer un peu de prolonger l'expérience. C'est vrai que ce n'était plus trop l'envie de traverser, d'aller en Europe qui nous motivait parce qu'avec ce que nous avons vécu, les expériences que nous avons partagées avec d'autres, on a commencé à comprendre que même ici en Afrique il pouvait y avoir des opportunités, on s'est dit : on va essayer de prolonger un peu l'expérience ici en Afrique " (Guillaume, Nouadhibou, décembre 2006).

Ils restent un temps à Bamako, entendent parler d'opportunités de travail en Mauritanie, arrivent à Nouakchott en 2001, pour y trouver une communauté ivoirienne bien organisée. Son ami " a la chance " d'obtenir un visa pour le Maroc, il passera plus tard en Espagne. De son côté, il vient à Nouadhibou pour se lancer dans le commerce du guedj à Bûntya, avant d'estimer au bout d'un an qu'il n'y trouve pas son compte, tant du point de vue financier que de celui de l'activité elle-même. Il tente, non sans angoisse, de maintenir le sens qu'a pour lui le choix de migrer :

" Je me suis dit que si j'arrivais en Europe je pourrais continuer mes études plus facilement peut-être, et en cours de route, j'ai peut-être changé d'idée [...], ça fait six années, si je retourne, par rapport aux objectifs que je m'étais fixés, j'ai un sentiment d'échec, pour moi qui n'ai connu que des succès, dans la vie scolaire, j'ai rarement, très peu échoué, je me dis il faut attendre, j'essaie de repousser... " (Guillaume, Nouadhibou, décembre 2006).

II essaie de l'expliquer à ses parents, à qui il ne peut envoyer d'argent. II redoute qu'ils puissent croire qu'il va les abandonner sans être sûr d'arriver à les convaincre. II s'entête donc et sa position à la mission prend alors un tour nouveau, à mesure que l'action caritative se développe avec la " crise humanitaire " entrainée par les départs massifs de pirogues. La mission de Nouadhibou est le centre où convergent les humanitaires. De son côté I'ONG catholique Caritas, qui depuis des années essaye de développer les associations nationales d'étrangers et de les regrouper dans une Union Générale, renforce ses programmes, rénove et agrandit ses locaux, devenus un foyer de formation et de loisirs ainsi qu'un lieu de réunion pour les migrants. Guillaume est la personne toute désignée, par son niveau d'éducation, sa bonne connaissance de première main de l'univers des aventuriers et aussi, par sa foi sincère qui le met en harmonie avec la philosophie sous-jacente qui inspire les actions, pour occuper de nouvelles responsabilités. II assure désormais les fonctions effectives de secrétaire général des projets d'appui aux migrants. Au-delà de la tenue des ordres du jour et des comptes-rendus, il est amené à jouer un rôle stratégique dans l'arbitrage des conflits et des orientations des différentes communautés. La mission est véritablement devenue son monde, un monde branché sur les logiques globalisées de l'action humanitaire conjuguées avec I'universalisme chrétien, mais aussi un monde où sa vocation pour les études trouve, avec la lecture des textes saints et l'approfondissement de sa culture religieuse, un nouvel exutoire. Guillaume tend ainsi à devenir une sorte de diacre, peut-être pas officiellement, mais en pratique. Lors de l'absence des prêtres, il préside à I'office dominical, faisant la lecture des Saintes Écritures et renvoyant en paix les fidèles. Dans le cadre de ses fonctions à la mission, il voyage et a participé, comme membre du réseau Caritas, à plusieurs forums sociaux où il a rencontré les représentants d'associations de migrants subsahariens d'autres pays " de transit". 
Cette position, à ses yeux comme à ceux de sa famille, va donner un sens nouveau à sa migration et à la définition de sa réussite. Sans plus redouter l'échec, il peut retourner, en vacances dans sa famille pour constater, non sans étonnement que tout lui indique qu'il est devenu un émigré, bien que démuni des signes de richesse que l'on attend d'eux. II raconte alors, avec émotion, qu'il a pu, au village, réunir un grand nombre des membres de sa famille élargie, et comment devant cette assemblée, il a dirigé la prière, célébrant son retour provisoire et remerciant Dieu ; confirmant ainsi son statut de lettré et d'homme religieux et la place nouvelle qu'elle lui donne parmi eux.

Quoiqu'assez satisfait apparemment de la position acquise à Nouadhibou (pas forcément financière précise-t-il) où il a passé plus de dix ans, il pense qu'un étranger ne peut jamais s'y intégrer totalement, on lui fait toujours sentir qu'il est un étranger. Tout peut se passer à tout moment. II pense au retour, mais est bien obligé de constater que cette même position d'étranger, qui rend son maintien à Nouadhibou de plus en plus problématique, est précisément celle qui lui confère le prestige dont il jouit auprès des siens.

Pour autant, Nouadhibou reste perçue comme un lieu d'échouage qu'il est prêt à quitter à tout moment si se présentent $d^{\prime}$ autres opportunités de trouver " ce qu'on a toujours souhaité ".

"Dans l'immédiat on est encore là, en espérant que dans l'avenir les choses seront plus favorables, d'obtenir ce qu'on a toujours souhaité [...]. On se rend compte que c'est le fait d'avoir visé l'Europe qui a fait qu'on n'est pas vraiment... nous, on avait une seule idée en tête, on n'a pas trop cherché, avant l'Europe qu'est-ce qu'y a ? Peut-être y a d'autres opportunités en Afrique australe... " (Guillaume, Nouadhibou, juillet 2013).

\section{Du refoulé au notable associatif, la professionnalisation de l'aventure à Bamako}

En contrepoint de ces trajectoires individuelles d'après transit, la configuration très différente de la situation à Bamako offre d'autres occasions de convertir l'expérience de la migration de transit en capital social, passant dans ce cas par la mobilisation collective.

\section{Faire entendre la voix des sans voix : le champ associatif à Bamako}

Contrairement à la Mauritanie, c'est autour de la question des migrants nationaux que s'est construite au Mali la problématique de la migration irrégulière. L'Association des Maliens Expulsés (AME) créée dès 1996 à l'initiative d'un expulsé d'Angola s'est rapidement imposée comme une instance de mobilisation des expulsés et d'interpellation à l'égard du pouvoir malien. L'association a su nouer des connexions et trouver des supports (syndicats et organisations de défense des immigrés) en ouvrant, autour de la question des " sans-papiers" et des " expulsés ", un champ migratoire entre la France et le Mali. Les revendications centrales, comme la récupération des biens et cotisations s'adressent à l'État français tout en donnant simultanément matière à un positionnement dans I'espace national malien (avec notamment le soutien du député de l'opposition Oumar Mariko). L'association s'affirme comme un groupe de pression à l'égard 
de l'État malien dans sa gestion des relations interétatiques liées à la migration, en faisant campagne contre la signature des accords de réadmission, présentés comme une honte nationale.

Lorsqu'au milieu des années 2000, les migrants refoulés d'Algérie dans leur tentative de passer en Europe commencent à se regrouper à Bamako et à s'organiser selon la formule éprouvée du " ghetto " (Laacher, 2007 ; Pian, 2008), ils trouvent sur place un champ associatif sur la migration irrégulière très actif au sein duquel ils vont devoir négocier leur place dans l'espace public local.

Sous l'impulsion de deux frères camerounais, arrivés à Bamako en 2006, I'Association des Refoulés d'Afrique Centrale au Mali (ARACEM) va négocier les termes dans lesquels se formule leur problème avec un ensemble d'autres acteurs (ONG internationales et associations locales, organismes internationaux, acteurs politiques) intervenant dans les débats, les choix politiques et la mise en œuvre des actions publiques dans le domaine des migrations.

La mobilisation se structure autour de la solidarité entre les résidents du ghetto, Camerounais, Congolais, Tchadiens, Centrafricains, expulsés d'Algérie ou de Libye, qui constituent une communauté d'infortune à la fois fluctuante et soudée, organisée autour de pratiques collectives de survie (collecte de nourriture, partage du loyer, solidarité dans le cas de maladies et de décès, visites aux migrants incarcérés à la prison) et d'embryons d'organisation collective pour le travail (salon de coiffure, vente de crédit téléphonique) sous forme de coopérative. La centralité urbaine du ghetto les met au contact de voisins maliens avec qui ils s'entendent bien (" Quand il n'y pas de quoi acheter à manger, je vais chez Traoré, il me dit: "il y a un plat pour toi" "). Par contre ils n'ont aucun contact avec les compatriotes résidents, commerçants ou étudiants qui, nous disent-ils, te regardent d'un autre œil, "comme des bandits ", peu de contacts avec la famille "à cause de la honte ". Ils vivent " entre voyageurs" ("On a les mêmes histoires à se raconter $")^{14}$.

Parallèlement aux activités de survie, ils mettent rapidement sur pied des activités de gestion d'image, tournées vers l'extérieur, visant à démentir la suspicion de délinquance volontiers associée à "l'aventurier ", par des actions citoyennes (nettoyage des canaux, distribution de dons à un village d'enfants SOS) qu'ils publicisent sur leur site Internet, nouvellement créé et sur les ondes d'une petite radio locale où ils animent une émission dénommée "Rêve incertain " consacrée aux problèmes des migrants en transit :

" [...] Nous essayerons de par cette prise d'antenne de faire comprendre et savoir au public, aux auditeurs, à la population et à tous ceux qui nous écouteront que nous pouvons également être utiles à la société, que nous ne sommes pas des malfaiteurs, mais de simples et dignes voyageurs victimes des politiques du Nord qui ont fait de nous des clandestins en s'opposant à la libre circulation des personnes, mais restant ouverts à celle des biens ${ }^{15}$.

14 Toutes les expressions en italique ont été recueillies lors de discussions collectives avec les résidents du ghetto menées en 2008.

15 ARACEM, Émission radio du 09 août 2008, [en ligne] consulté le 24/02/2014. URL : http://aracem.canalblog.com/archives/2008/08/09/10184899.html 
Les efforts pour récuser l'étiquette de clandestin n'impliquent pas pour autant la revendication d'un statut d'immigré, comme en témoigne leur refus obstiné de demander une carte de résident, en dépit des pressions exercées par leurs soutiens locaux. Leurs revendications ne portent pas sur une demande d'insertion dans la société locale, mais sur la reconnaissance d'un droit à être là, en transit ou comme ils préfèrent le dire en parcours.

Si I'auto-désignation " dignes voyageurs ", présente dans les slogans et affiches de l'association, est préférée à celle d' "aventurier ", toujours susceptible d'être employée négativement, ils n'en revendiquent pas moins l'aventure comme une modalité de construction de leur identité et de la légitimité de leur quête. Au-delà des actions de solidarité et d'entraide entre refoulés, les deux leaders de I'ARACEM se livrent ainsi à un véritable travail d'entrepreneurs d'identité, qui place la distribution inégale du droit à la mobilité au cœur de la dénonciation de l'injustice. Ils tentent ce faisant d'imposer une image des " migrants de l'aventure " comme des individus porteurs de droits humains universels, dont celui qui donne sens à leur expérience : le droit à la circulation.

Ce langage de la reconnaissance s'affirme comme une résistance à l'imposition de sédentarité et à la délégitimation de l'aventure par les organismes de gestion de l'immigration dont les migrants perçoivent clairement la violence symbolique. II s'affirme comme une tentative d'opposer un contre discours autonome à ceux qui prétendre dire "leur vérité " sur ces mobilités : selon des versions alternativement misérabiliste (lorsque l'accent est mis sur les drames des noyades), criminalisante (lorsqu'il est mis sur les " trafics d'êtres humains "), ou pathologisante (lorsqu'il est mis sur la folie de la ruée suicidaire vers l'eldorado).

Mais il se démarque aussi d'emblée fortement du langage et du répertoire d'actions des expulsés nationaux. L'arrimage de l'AME aux organisations soutenant en France la lutte des travailleurs sans papiers (Ia CGT, Droit devant, le Réseau Éducation Sans Frontières), ainsi que son ancrage dans le jeu politique malien impliquent de porter sur la migration un regard tenant compte des intérêts du pays et se démarquant de l'idéologie de l'aventure.

"La sensibilisation, c'est un programme que nous avons mis en marche depuis 2007, c'est la démystification du mieux-être ailleurs, et la valorisation de l'individu dans son milieu local. Sur un point de défense des droits humains, nous défendons la libre circulation, mais dans le contexte de la convention internationale de la protection des droits des migrants. Qu'ils soient sans papiers, nous les défendons là-bas, et nous avons les moyens

de les défendre même en France et en Espagne, ça, c'est la transnationalisation de la lutte. Mais d'un autre côté, nous en Afrique, nous avons notre particularité, nous avons la spécificité malienne et partant de là, nous voyons que sur un an, c'est nous qui perdons assez de frères, dans la mer, dans le désert, donc pour avoir la légitimité d'interpeller nos autorités, il faut mettre en place un programme de sensibilisation qui puisse permettre à ces jeunes, pas de briser l'envie d'aventure, mais de les réintéresser au local. Quand on dit : ouvrez les frontières, ce n'est pas pour dire à tous nos enfants, à nos frères : partez! Non, ce n'est pas ça, nous avons besoin de nous-mêmes pour construire notre pays..." (président de l'AME, Bamako, février 2009). 
Les migrants refoulés lors des tentatives de passage des frontières, qu'ils soient maliens ou étrangers, se sont organisés en dehors de I'AME. Ils sont pour ainsi dire abandonnés aux petites associations (ARACEM, ARTD ${ }^{16}$ ) qui tentent de se faire une place dans un marché de l'assistance aux migrants où les subsides se redistribuent en cascade depuis les grosses ONG elles-mêmes pourvoyeuses de ressources $\left(\mathrm{CIMADE}^{17}\right.$, Caritas, Medico International, Médecins du Monde, $\mathrm{CISP}^{18}$ ), jusqu'aux associations locales qui dépendent des premières pour I'octroi de subventions, mais leur sont aussi indispensables comme relais pour la connaissance du terrain.

La mise en place fin 2008 du Centre d'Information et de Gestion des Migrations (CIGEM) va profondément modifier la donne dans ce petit milieu associatif intervenant sur les questions de migration. Financé par le Fonds Européen pour le Développement de la Commission européenne (FED), le CIGEM a pour objectif d'inscrire la problématique de la migration irrégulière (des actions, des publics et des objectifs) dans le cadre de I' " approche globale " (élaboration d'une politique migratoire, lutte contre la migration clandestine, développement), ciblant comme bénéficiaires de ses subventions les associations intervenant aussi bien auprès des migrants potentiels, des migrants de retour volontaire ou involontaire et des migrants en transit.

L'unification sous l'égide du CIGEM du secteur d'études et d'intervention auprès des migrants (assistance, campagnes de dissuasion contre l'immigration clandestine, réinsertion, formation) va se traduire par l'arasement des revendications particularistes (des droits des expulsés, de la réinsertion des refoulés, de la reconnaissance des migrants de transit) et des distinctions nationales ou régionales (Maliens, Afrique centrale) telles qu'elles s'étaient constituées par les associations spontanées des migrants, au profit de l'assistance aux " retournés forcés "(Maliens expulsés de France, Maliens et autres Africains refoulés du Maroc, d'Algérie et de Mauritanie, rapatriés de Côte d'Ivoire, puis des réfugiés de Libye à partir de 2011 et des déplacés du Nord en 2012) plaçant toutes les associations en concurrence dans un marché considérablement élargi.

En même temps qu'elle tend à unifier les publics et les domaines d'intervention, cette restructuration du champ associatif tend à polariser les intervenants selon d'autres principes de différentiation, notamment en termes de morale politique se traduisant par des choix d'alignement : être du côté des instances de la gestion migratoire (CIGEM-UE-OIM, etc.) ou du côté des "retournés forcés" et de leurs soutiens (CIMADE, Médecins du Monde, Medico International, AMNESTY Mali, AMDH, etc.).

Cette recomposition a redistribué les cartes en fonction des capacités plus ou moins grandes d'adapter les lignes d'action de départ au nouveau contexte.

16 Association Retour, Travail, Dignité, créée par des Maliens refoulés.

17 Comité inter-mouvements auprès des évacués.

18 Comitato Internazionale per lo Sviluppo dei Popoli : présente en Algérie, au Maroc, en Tunisie, au Niger, au Mali et au Congo, l'association œuvre pour la prévention de I'immigration clandestine et mène des actions d'incitation au retour volontaire par le financement de micro-crédits. 
Une association comme I'ARTD dont le créneau d'intervention en tant que représentant des nationaux maliens refoulés est celui de la réinsertion, a tenté de négocier une aide à l'installation (achats de matériel agricole, attribution de parcelles) contre l'enrôlement dans les campagnes de dissuasion contre la migration irrégulière. Leur expérience de refoulé leur permettant de porter une parole de l'intérieur d'une communauté de souffrance répond bien à la demande des institutions et ils auraient pu constituer le partenaire idéal en donnant corps à une figure du repenti, qui a fini par prendre conscience de son erreur et est disposé à ramener les autres dans le droit chemin. Mais cela rend simultanément la revendication de réinsertion en tant que citoyens maliens fragile car la théorie de l' " appel d'air " chère aux experts du Migration Management répugne à faire reconnaître un privilège par rapport à ceux qui ne sont pas partis. Étant par ailleurs les plus démunis en ressources (culturelles, linguistiques, scolaires) permettant de se réapproprier le langage des ONG, ils ne sont pas de taille à lutter contre les " associations cartable " ${ }^{19}$ qui se sont introduites dans le marché, tout en se trouvant par ailleurs stigmatisés comme "vendus " par les associations militantes.

L'AME est prise dans d'autres contradictions : I'élargissement de ses activités traditionnelles (accueil à l'aéroport, information et aide juridique des expulsés, recueil de témoignages) à des fonctions plus proches du travail social (hébergement, "assistance psycho-médicale ") qui occupent une place de plus en plus importante dans le marché élargi, risque de l'amener à assumer des services que l'État n'assure pas et s'en faire ainsi volens nolens un auxiliaire à qui on laisse le soin d'arrondir les angles. Autrement dit, un risque de dépolitisation de son action alors que I'AME se veut une force politique.

\section{De la solidarité du ghetto à l'institutionnalisation de l'accueil}

Les deux leaders de I'ARACEM ont totalement réussi à tirer leur épingle du jeu en grande partie du fait de leur position d'étranger, qui leur permet d'éviter les contradictions et les systèmes de double contrainte qu'affrontent les associations de nationaux.

Lorsque nous les retrouvons en 2011, l'un d'eux est sur le point de partir le soir même à la frontière Libye-Égypte, avec Médecins du Monde qui est devenu leur partenaire dans le cadre d'un projet d'appui psychologique aux refoulés. Avec l'élargissement de leur domaine d'intervention à tous les migrants de retour forcé, étrangers et Maliens, les partenaires se sont multipliés : le PNUD ${ }^{20}$, Médecins du Monde, Medico international Allemagne, la CIMADE, tandis qu'ils jouissent d'une pleine reconnaissance dans le réseau transnational de l'altermondialisme : Fortress Europ, Migreurop, AfricEuroplnteract, qui les désigne comme des invités légitimes aux forums sociaux mondiaux, et leur permet aussi de voyager en dehors de I'Afrique : en Turquie et à Paris en 2012, à Bruxelles en 2013, à Berlin en 2014.

19 Associations constituées en vue de capter des fonds publics, dont l'existence se résume à une boîte postale et au " cartable " de leur promoteur contenant les " projets " destinés à attirer ces fonds.

20 Programme des Nations Unies pour le Développement. 
En quelques années le petit regroupement d'aventuriers lié au ghetto qui mendiait les restes des subsides dispensés par les ONG, est devenu l'une des plus importantes associations de migrants au Mali, aspirant à faire jeu égal avec I'AME. Devenus partenaires des deux gros projets dont bénéficiait depuis le début I'AME ${ }^{21}$, les fondateurs de I'ARACEM sont maintenant des notables, incontournables dans le milieu des organismes locaux liés à la migration, et des points de passage obligés pour les ONG qui développent des programmes d'assistance aux migrants irréguliers. Ils ont déménagé dans un nouveau local, une vaste villa dont le loyer est payé par répartition entre leurs différents partenaires. Le changement n'est pas que dans la surface et le confort accru, il manifeste la privatisation du collectif que représentait le ghetto :

"Au ghetto, le chef était chef c'est vrai, mais ses pouvoirs s'étendaient vraiment pas, tu pouvais pas avoir la décision sur quelqu'un parce qu'il te disait: "Ici c'est pas ta maison" " (entretien avec les responsables de I'ARACEM, Bamako, mars 2011).

C'est bien observé. L'institutionnalisation de l'accueil, en conformité avec les normes occidentales de I'humanitaire (Lecadet, 2011 : 291), sape I'autorité reconnue au chef de ghetto comme figure charismatique de l'auto-organisation au profit d'une source exogène de légitimité qui confère aux leaders reconnus par les institutions leur appropriation des lieux et leur pouvoir sur les résidents.

Au cours de ce processus de professionnalisation, la relation de solidarité entre " camarades " s'est métamorphosée en une relation entre aidants et aidés, voire entre soignants et soignés : dans le cadre du programme de Médecins du Monde sur la santé mentale qui constitue désormais une part essentielle de leurs ressources, ils sont chargés d'établir une nosographie sommaire des cas de traumatisme, et de la prise en charge des cas les moins graves à travers l'animation de groupes de parole, technique pour laquelle ils ont reçu une formation spécifique.

Si les trajectoires individuelles des deux leaders de I'ARACEM s'ancrent dans I'engagement collectif, elles soulignent paradoxalement la difficulté qu'ont ces migrants à se faire reconnaître collectivement sur la scène sociale et politique. Les mobilités de l'aventure expriment incontestablement une part de protestation contre (et des formes de résistance à) une domination, elles ne se prêtent guère, toutefois, à la mobilisation protestataire. D'une part elles sont portées par une idéologie fortement individualiste (" chacun sa chance ") qui n'empêche pas la reconnaissance mutuelle entre des gens qui partagent cette idéologie et ont des intérêts communs, mais confine l'action collective aux coopérations ponctuelles et orientées vers un but à court terme (franchissement des barrières, organisation de la survie dans les ghettos). D'autre part, l'alignement sur les agendas et les lignes d'action des organismes financeurs normalisent les revendications dans un langage bureaucratique, technique ou militant : la dimension rebelle de la protestation se trouve disciplinée sous la forme du "plaidoyer ", la violence du contrôle des frontières est recadrée dans le registre médical des

21 Le projet Médecins du Monde : “Améliorer l'accès aux soins en santé mentale pour les personnes migrantes en situation de retour forcé au Mali " et le projet régional Défense des droits des migrants dans les pays de transit (Algérie, Maroc, Mauritanie, Niger et Sénégal) de la CIMADE financé principalement par le ministère des Affaires étrangères. 
techniques de prise en charge de la souffrance des traumatisés. Recodées en langage bureaucratique, les compétences spécifiques (le lien avec le milieu des aventuriers, la connaissance de première main du " terrain ") qui font de ces migrants des partenaires potentiels pour les institutions (étatiques, internationales, humanitaires, associatives) impliquent une forme plus ou moins sincère ou intéressée d'adhésion à leur discours qui peut susciter la méfiance de la part de ceux qu'on prétend représenter.

La reconnaissance institutionnelle des collectifs spontanés formés par les " migrants de transit ", permet d'échapper à la place subordonnée concédée aux acteurs faibles (Payet et al., 2008) et de faire émerger une petite élite de notables associatifs, au prix cependant d'un éloignement des compréhensions partagées qui rendent ces mobilités de l' " aventure " socialement et moralement légitimes aux yeux de leurs acteurs : la migration comme aventure, revendiquée comme épopée héroïque de celui qui " cherche sa chance "; comme héritage d'une tradition qui fait du séjour provisoire dans un univers étranger un rituel de passage à l'âge d'homme ; ou comme devoir familial qui commande d'aller chercher où elles se trouvent les ressources permettant de s'acquitter de sa dette sociale.

\section{Conclusion}

Pour différents qu'ils soient, ces itinéraires mettent tous en scène de nouvelles figures des mobilités contemporaines qui ont cessé de s'inscrire dans la problématique de l'émigré/immigré (Sayad, 1992) sans pour autant relever des formes de migration dites "transnationales ", ce terme faisant référence aux engagements simultanés et aux liens multiples (politiques, économiques, culturels) que les migrants entretiennent avec leurs pays d'origine et leurs pays de résidence (Glick Schiller et al., 1995). Du fait de leur ancrage local qui les fixe dans le lieu du transit, leurs parcours ne peuvent pas non plus se confondre avec ceux des transmigrants de l'économie souterraine qui s'inscrivent, par leurs pratiques incessantes de va-et-vient, dans le paradigme de la circulation et de la mobilité (Tarrius, 2002).

En effet, d'une part l'expérience de "l'entre-deux " vécue par ces migrants ne saurait être confondue avec celle de la double absence vécue par les immigrants algériens. Dans leur cas, le prolongement de la vie sur la frontière vient brouiller la division entre pays de départ et pays d'accueil. Le provisoire qui dure, l'incertitude de l'avenir ne renvoient pas à un choix difficile entre les deux pôles d'ici et de là-bas, mais à l'ambiguïté des situations liminaires (Turner, 1990).

D'autre part, la dimension incontestablement transnationale de ces migrations, ne revient pas tant à se mouvoir dans deux lieux de vie (et deux espaces de citoyenneté) qu'à se connecter aux différents réseaux déterritorialisés (religieux, commerçants, associatifs) se croisant dans les lieux de transit. En se branchant sur ces réseaux extérieurs qui protègent, quoique de façon relative, des contraintes exercées par les pouvoirs locaux, les migrants peuvent mettre à profit les micro-bénéfices engrangés en chemin et l'auto-formation acquise dans 
I'expérience de l'aventure ${ }^{22}$. La vie sur la frontière offre ainsi aux plus chanceux ou aux plus astucieux de ces laissés pour compte de l'aventure l'opportunité de tirer leur épingle du jeu, alors qu'avec la crise en Espagne, ceux qui avaient réussi l'épreuve du passage risquent paradoxalement de se retrouver du côté des perdants.

Ces différents parcours témoignent des capacités d'initiative des migrants. Certes, l'attention portée aux individus, inévitable dans des études de cas portant sur des modifications de trajectoires, amène, par construction, à mettre en exergue leurs qualités personnelles, leur astuce, leur ténacité, leur courage. II est donc particulièrement important de souligner leur caractère situé avec ce qu'elles doivent au contexte dans lequel elles se manifestent. Ces qualités requises pour pérenniser ce fragment de l'existence isolé du flux de la vie, détaché du passé et sans lien avec l'avenir que représente l'aventure (Simmel, 2002), doivent ainsi se comprendre en regard des conditions qu'imposent les politiques de contrôle des mobilités et de mise à l'écart des indésirables. Les cas étudiés, cependant, ne sauraient être assimilables aux situations décrites par Agier (2002) selon qui des fractions de plus en plus importantes de populations du Sud sont amenées à vivre leur existence " aux bords du monde ". La prolongation indéfinie de la parenthèse, que représente pour les aventuriers la vie sur la frontière, est le corrélat des capacités, acquises dans le cours même de l'expérience migratoire, à vivre de la frontière. Dans leur cas, ces capacités ne favorisent pas le retour, comme cela peut être le cas pour d'autres types de migrants (Beauchemin et al., 2013). Investies sur place, elles les ancrent localement, tout en maintenant ouverte leur expérience migratoire à de possibles développements futurs. Aussi se maintiennent-ils dans une position d'étranger. Étrangers, ces migrants le sont à double titre : par leur extériorité à la société locale et à ses normes culturelles, sociales et politiques, par choix ou mise à distance ; mais aussi par leur réussite même qui prend appui sur les espaces résiduels et les interstices des économies locales concédés aux étrangers. C'est en investissant un secteur productif (la transformation du poisson) dédaigné par les opérateurs locaux qu'Adam s'est construit une carrière de petit entrepreneur ; c'est en se taillant une place dans un dispositif transnational catholique ne pouvant guère être disputée par les nationaux dans un pays $100 \%$ musulman, que Guillaume est sorti " grandi " de l'épreuve du seuil ; c'est en prenant pour base ce lieu par excellence de l'extranéité que représente le ghetto que les deux frères camerounais fondateurs de I'ARACEM ont su conquérir, en convertissant l'expérience de l'aventure en compétence dans la gestion des migrations, la place sociale qu'ils avaient auparavant espérée trouver dans une hypothétique carrière de footballeur en Europe.

Enfin, l'extériorité maintenue aux communautés et aux lieux d'origine permet de se libérer des contraintes inhérentes aux chaînes de dépendance domestiques, voire d'en réaménager à distance l'ordre social. Mais cet ancrage local et ce réaménagement des relations avec le pays d'origine, ne doivent pas, comme nous l'avons souligné, être confondus avec la situation caractéristique

22 Dont on peut supposer néanmoins qu'elle peut d'autant plus être mise à profit qu'elle s'appuie sur un capital scolaire acquis avant la migration, ce qui est le cas des migrants dont nous avons ici présenté la trajectoire, mais aussi de tous les autres cas de réussite dans le post-transit que nous avons eu I'occasion d'observer. 
de "l'immigré ", car cet " ailleurs ", que tend à devenir le pays des origines, n'a aucun " ici " pour contrepartie. L'installation dans la ville de transit ne se présente jamais comme un choix de vie, et même après des années de présence et l'abandon définitif du projet d'Europe, le lieu de l'ancrage reste néanmoins vécu comme un espace liminaire, un seuil qui n'est plus borné par le franchissement de la frontière, mais par la promesse toujours ouverte et à venir d'une bonne vie ailleurs. En témoigne dans les récits la perspective d'un redéploiement vers d'autres horizons (I'Afrique du Sud, voire pour certains la Chine) qui maintient l'imaginaire du voyage.

\section{Références bibliographiques}

Agier Michel (2002) Aux bords du monde, les réfugiés, Paris, Flammarion, 187 p.

Alioua Mehdi (2005) La migration transnationale des Africains subsahariens au Maghreb. L'exemple de l'étape marocaine, Maghreb-Machrek, 185, pp. 37-58.

Ba Cheikh Oumar et Choplin Armelle (2005) Tenter I'aventure par la Mauritanie: migrations transsahariennes et recompositions urbaines, Autrepart, 4 (36), pp. 21-42.

Bakewell Oliver (2008) "Keeping them in their place": the ambivalent relationship between development and migration in Africa, Third World Quaterly, 29 (7), pp. $1341-1358$.

Bauman Zigmunt (1999) Le coût humain de la mondialisation, Paris, Hachette Littératures, $180 \mathrm{p}$.

Bayart Jean François (1989) L'État en Afrique. La politique du ventre, Paris Fayard, $439 \mathrm{p}$.

Beauchemin Cris, Kabbanji Lama, Sakho Papa et Schoumaker Bruno (Éds.) (2013) Migrations africaines : le co-développement en questions, Paris, Armand Colin, $343 \mathrm{p}$.

Bredeloup Sylvie (2008) L'aventurier une figure de la migration africaine, Cahiers Internationaux de Sociologie, 125 (2), pp. 281-306.

Bredeloup Sylvie (1994) L'aventure contemporaine des diamantaires sénégalais, Politique Africaine, 56, pp. 77-93.

Choplin Armelle (2010) "Quand la mer se ferme ". Du transit au post-transit migratoire en Mauritanie, Hommes et Migrations, 1286-1287, pp. 74-95.

Escoffier Claire (2008) Transmigrant-e-s africain-e-s au Maghreb, Paris L'Harmattan, $210 \mathrm{p}$.

Glick Schiller Nina, Basch Linda and Szanton Blanc Cristina (1995) From Immigrant to Transmigrant: Theorizing Transnational Migration, Anthropological Quarterly, 68 (1), pp. 48-63.

Goldschmidt Elie (2002) Migrants congolais en route vers I'Europe, Les Temps Modernes, 620-621, pp. 208-239.

Laacher Smaïn (2007) Le peuple des clandestins, Paris, Calmann-Lévy, 215 p. 
Lecadet Clara (2011) Le front mouvant des expulsés. Lieux et enjeux des regroupements et des mobilisations collectives des migrants expulsés au Mali, Thèse d'anthropologie sous la direction de Michel Agier, Paris, EHESS.

Marie Alain (1997) L'Afrique des individus, Paris, Karthala, 436 p.

Payet Jean-Paul, Giuliani Frédérique et Laforgue Denis (2008) La voix des acteurs faibles. De l'indignité à la reconnaissance, Rennes, Presses Universitaires de Rennes, $246 \mathrm{p}$.

Pian Anaik (2008) Aux portes de Ceuta et Melilla : regard sociologique sur les campements informels de Bel Younes et de Gourougou, Migrations Sociétés, 20 (116), pp. 12-24.

Poutignat Philippe (2011) Migration at the level of individuals, in Jocelyne StreiffFénart and Aurélia Segatti Eds., The Challenge of the Treshold: Border Closures and Migration Movements in Africa, Maryland, Lexington Books, pp. 177-196.

Poutignat Philippe et Streiff-Fénart Jocelyne (2010) Migration Policy Development in Mauritania: Process, Issues, and Actors, in Martin Geiger and Antoine Pécoud Eds., The Politics of International Migration Management, Basingstoke, Palgrave Macmillan, pp. 202-219.

Poutignat Philippe et Streiff-Fénart Jocelyne (2006) De I'aventurier au commerçant transnational, trajectoires croisées et lieux intermédiaires à Nouadhibou (Mauritanie), Cahiers de la Méditerranée, 73, pp. 129-149.

Sayad Abdelmalek (1992) L'immigration ou les paradoxes de l'altérité, Bruxelles, De Boeck University, $331 \mathrm{p}$.

Sayad Abdelmalek (1977) Les trois " âges " de l'émigration algérienne en France, Actes de la recherche en sciences sociales, 15 (1), pp. 59-79.

Simmel Georg (2002) La philosophie de l'aventure, Paris, L'Arche éditeur, 120 p.

Streiff-Fénart Jocelyne (2011) The manufacture of transit, in Jocelyne StreiffFénart and Aurélia Segatti Eds., The Challenge of the Treshold: Border Closures and Migration Movements in Africa, Maryland, Lexington Books, pp. 39-55.

Streiff-Fénart Jocelyne et Poutignat Philippe (2008) Nouadhibou "ville de transit " ? Le rapport d'une ville à ses étrangers dans le contexte des politiques de contrôle des frontières de l'Europe, Revue Européenne des Migrations Internationales, 24 (2), pp. 193-217.

Streiff-Fénart Jocelyne and Segatti Aurelia (Eds.) (2011) The Challenge of the Treshold: Border Closures and Migration Movements in Africa, Maryland, Lexington Books, 269 p.

Tarrius Alain (2002) La mondialisation par le bas, Paris, Balland, 168 p.

Timera Mahamet (2009) Aventuriers ou orphelins de la migration internationale? Nouveaux et anciens migrants subsahariens au Maroc, Politique Africaine, 115, pp. 175-195.

Turner Victor (1990) Le Phénomène rituel. Structure et contre structure, Paris, PUF, 206 p. 


\section{Jocelyne Streiff-Fénart et Philippe Poutignat}

\section{... Vivre sur, vivre de la frontière: l'après transit en Mauritanie et au Mali}

Cet article porte sur la situation des migrants " de transit " qui sont restés dans le pays traversé. II prend comme lieux d'observation les villes de Nouadhibou en Mauritanie et Bamako au Mali. Trois études de cas présentent des façons très différentes de convertir l'expérience de la migration en capital social et de tirer profit de la vie sur la frontière. Elles ont cependant en commun le branchement sur des réseaux transnationaux. À Nouadhibou, un dispositif de commerce à longue distance et un réseau religieux ; à Bamako, la nébuleuse des organismes de gestion, d'accueil et d'assistance aux migrants. Dans ce dernier cas, en contrepoint des trajectoires individuelles exposées en premier lieu, les auteurs proposent l'analyse d'une mobilisation collective formée autour de la solidarité entre résidents du " ghetto " des " refoulés d'Afrique Centrale ". Ils examinent tout particulièrement son insertion dans le champ associatif sur la migration irrégulière.

\section{Living on the Frontier: Post-transit in Mauritania and Mali}

This article focuses on the situation of "transit" migrants remaining in the country of transit. It is based on a long-term observation study of Nouadhibou (Mauritania) and Bamako (Mali). Three case studies reveal diverging ways of transforming migration experiences into social capital, while taking advantage of the opportunities offered by life on the frontier. All cases have in common to be connected with transnational networks. These include for instance international trade and religious network in Nouadhibou or a heterogeneous network of migration management and humanitarian aid organizations in Bamako. In the latter, as a counterpoint to the emphasis placed on individual trajectories, the authors offer an account of collective mobilization and links of solidarity within the ghetto of the so-called "Central Africa deportees", with a specific focus on its integration into the existing network of illegal migrant associations.

\section{Vivir en, vivir de la frontera: el postránsito en Mauritania y Mali}

Este artículo trata de la situación de los emigrantes "de tránsito", de los que se quedaron en los países de tránsito hacia su destino final. Toma como punto de referencia para la observación las ciudades de Nouadhibou, en Mauritania, y Bamako, en Mali. A través del estudio de tres casos particulares se presentan tres modos diferentes de convertir la experiencia de la emigración en capital social, sacando provecho de este modo de vida en la frontera. Los tres casos tienen en común, con todo, su conexión con redes internacionales: en Nouadhibou, un dispositivo de comercio a larga distancia y una red religiosa; en Bamako, la nebulosa de los organismos de gestión, acogida y asistencia a emigrantes. En este último caso, y como contrapunto de las trayectorias personales anteriormente explicadas, se propone el análisis de la movilización colectiva en torno a la solidaridad conformada entre residentes del "gueto" y los "expulsados de África Central». Se examina así, particularmente, la inserción de estos últimos en el seno del ámbito asociativo sobre la emigración ilegal. 